\title{
Transcutaneous contrast-enhanced ultrasound imaging of the posttraumatic spinal cord
}

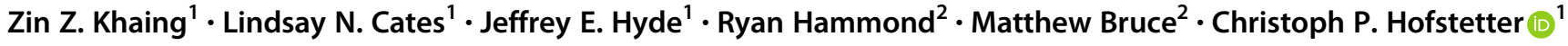

Received: 8 August 2019 / Revised: 21 November 2019 / Accepted: 9 December 2019 / Published online: 21 January 2020

(c) The Author(s), under exclusive licence to International Spinal Cord Society 2020

\begin{abstract}
Study design Experimental animal study.

Objective The current study aims to test whether the blood flow within the contused spinal cord can be assessed in a rodent model via the acoustic window of the laminectomy utilizing transcutaneous ultrasound.

Setting Department of Neurological Surgery, University of Washington, Seattle WA.

Methods Long-Evans rats $(n=12)$ were subjected to a traumatic thoracic spinal cord injury (SCI). Three days and 10 weeks after injury, animals underwent imaging of the contused spinal cord using ultrafast contrast-enhanced ultrasound with a Vantage ultrasound research system in combination with a $15 \mathrm{MHz}$ transducer. Lesion size and signal-to-noise ratios were estimated via transcutaneous, subcutaneous, or epidural ultrasound acquisition through the acoustic window created by the original laminectomy.

Results Following laminectomy, transcutaneous and subcutaneous contrast-enhanced ultrasound imaging allowed for assessment of perfusion and vascular flow in the contused rodent spinal cord. An average loss of $7.2 \mathrm{~dB}$ from transcutaneous to subcutaneous and the loss of $5.1 \mathrm{~dB}$ from subcutaneous to epidural imaging in signal-to-noise ratio (SNR) was observed. The hypoperfused injury center was measured transcutaneously, subcutaneously and epidurally $(5.78 \pm 0.86,5.91 \pm 0.53$, $5.65 \pm 1.07 \mathrm{~mm}^{2}$ ) at 3 days post injury. The same animals were reimaged again at 10 weeks following SCI, and the area of hypoperfusion had decreased significantly compared with the 3-day measurements detected via transcutaneous, subcutaneous, and epidural imaging respectively $\left(0.69 \pm 0.05,1.09 \pm 0.11,0.95 \pm 0.11 \mathrm{~mm}^{2}, p<0.001\right)$.

Conclusions Transcutaneous ultrasound allows for measurements and longitudinal monitoring of local hemodynamic changes in a rodent SCI model.
\end{abstract}

\section{Introduction}

Direct mechanical spinal cord tissue destruction caused by spinal trauma is often limited. However, subsequent edema, hemorrhage, and inflammation lead to a secondary wave of tissue damage. Peri-lesional edema, hemorrhage, and necrosis result in increased intraspinal pressure. Together with systemic hypotension due to the spinal shock, increased intraspinal pressure causes an almost

Christoph P. Hofstetter

chh9045@uw.edu

1 Department of Neurological Surgery, The University of Washington, Seattle, WA, USA

2 Applied Physics Laboratory, Center for Industrial and Medical Ultrasound, The University of Washington, Seattle, WA, USA complete loss of blood flow, also known as ischemia, in the injury center and significant hypoperfusion in the penumbral zone with progressive cell death overtime [13]. Two routinely performed clinical treatment strategies aim to improve local tissue perfusion of the contused spinal cord. First, surgical decompression of the spinal cord is recommended within $24 \mathrm{~h}$ of the initial injury [47]. Second, trauma guidelines recommend maintenance of the mean arterial blood pressure (MAP) at $85-90 \mathrm{Hg}$ for the first 7 days after acute spinal cord injury [8]. However, achieving a MAP target with fluid and vasopressor agent administration does not necessarily result in an optimal spinal cord perfusion pressure $[9,10]$. In fact, utilization of high-dose vasopressor agents with the intention to increase MAP may be associated with drug-related adverse events in some patients [11]. Therefore, there is a tremendous need for novel technologies to monitor local spinal cord hemodynamics. 
Several different strategies have been developed to monitor posttraumatic spinal cord hemodynamics. First, the spinal arterial perfusion pressure can be estimated by measuring the intraparenchymal spinal cord pressure at the injury site and subtracting it from the mean arterial pressure. Utilizing this approach decreased spinal cord perfusion pressures have been detected during the first week after a traumatic injury in both rodents [12] and patients [9]. The above invasive technologies require implantation of a sensor for hemodynamic measurements of the spinal cord $[9,13]$. Near infrared spectroscopy is another technology that has been utilized preclinically to measure tissue oxygen saturation of the contused spinal cord [14, 15]. It allows for real-time monitoring of hemodynamic changes and has been suggested to be more sensitive to ischemia-induced dysfunction than motor evoked potentials [16]. Lastly, ultrasound is another technology that has been utilized to determine hemodynamic changes of the traumatized rodent spinal cord $[17,18]$. The use of traditional ultrasound to image blood flow in the spinal cord of a rodent model was demonstrated by Soubeyrand et al. [17, 19]. Recently, our group has developed a novel high framerate and high frequency ultrafast contrast-enhanced ultrasound (CEUS) Doppler approach [20, 21]. Importantly, microbubbles give ultrasound the ability to determine flow velocity $(\sim \mathrm{mm} / \mathrm{s})$ blood flow in the microcirculation beyond the limitations of Doppler ultrasound. In all of these experiments from work done by our group as well as the Soubeyrand group, the ultrasound image acquisitions were obtained above the epidural membrane.

Here, we extend upon this work in investigating the ability of high frequency CEUS to follow blood flow changes in the spinal cord of a tSCI rodent model by imaging transcutaneously through paraspinal tissue via the acoustic window created by the surgical laminectomy. This approach allows for the noninvasive assessment of longitudinal changes in blood flow following tSCI in a rodent model.

\section{Materials and methods}

\section{Spinal cord injury}

Surgical procedures were performed according to an approved protocol following all appropriate guidelines from the University of Washington Institutional Animal Care and Use Committee. Two cohorts of six rats each were imaged at two different time points following contusion injury. The first cohort was imaged 3 days following injury, with the second cohort being imaged 10 weeks following injury. Female Long-Evans rats ( $n=12,250-300 \mathrm{~g}$ at the time of experiments; Envigo,
Indianapolis, IN) were utilized for our experiments. The rats were anesthetized using isoflurane (5\% to induce and $2.5-3 \%$ to maintain) and the area overlying the thoracic vertebral region was shaved, cleaned, and sterilized. A subcutaneous injection of bupivacaine and Lidocaine (1.5 $\mathrm{mg} / \mathrm{kg}$ ) was given prior to a longitudinal incision made overlying the T5-T11 area using a \#10 scalpel blade. The animals received perioperative subcutaneous lactated Ringer's solution (10 mL/kg/h), Gentamicin ( $5 \mathrm{mg} / \mathrm{kg})$, and Buprenorphine $(0.05 \mathrm{mg} / \mathrm{kg})$. A 24-gauge tail vein catheter was established and attached to a three-way stopcock for the introduction of microbubbles (Definity ${ }^{\circledR}$; Lantheus, NJ, USA) and subsequent saline ( $0.9 \%$ sodium chloride) flushes. After subperiostal dissection of paraspinal muscles, a laminectomy was performed to expose the spinal cord from $\mathrm{T} 7$ to T9. Animals were suspended at the T7 and T9 spinous process for the contusion injury. A 200 kDyn contusion injury was produced at T8 using an Infinite Horizon spinal cord contusion device (Precision Systems and Instrumentation, LLC). Acutely after injury, CEUS imaging was performed (see details below). At the end of the imaging session, the overlying muscles were sutured (Covidien, Polysorb ${ }^{\mathrm{TM}} 4-0$ ), and the skin was closed with staples. The animals were allowed to recover from anesthesia before being single housed and returned to the vivarium. During postoperative monitoring, the rats were health checked twice a day up to 14 days for the 10-week cohort. Manual bladder expression was carried out until bladder function normalized ( 14 days). The postsurgical treatment included subcutaneous Lactated Ringer's Solution when necessary to treat dehydration, buprenorphine $(0.05 \mathrm{mg} / \mathrm{kg}$, subcutaneous) to treat postoperative pain was given twice a day for 3 days, and gentamicin $(4.8-5 \mathrm{mg} / \mathrm{kg}$, subcutaneous) was given daily for 5 days for infection prophylaxis. Skin staples were removed after 10 days, and the animals were group housed.

\section{Ultrasound imaging technique}

For ultrasound imaging, the Vantage ultrasound research system (Verasonics, Seattle, USA) combined with a 15 $\mathrm{MHz}$ transducer (Vermon, Tours, France) was utilized. For each CEUS acquisition, a bolus of $0.1 \mathrm{~mL}$ of microbubbles was injected intravenously followed by a $0.2 \mathrm{~mL}$ saline flush. Ultrafast plane-wave acquisitions $(\sim 10,000$ frames/s) $[22,23]$ interleaved with a nonlinear pulsing sequence to remove tissue signal were collected which allows for assessment of perfusion and larger vasculature flow [21]. The nonlinear Doppler sequence enables the separation of both low velocity microbubbles in the microcirculation resulting in what we termed "perfusion" imaging and higher velocity microbubbles in the larger vasculature resulting in "vascular" imaging. 


\section{Contrast-enhanced ultrasound of SCI}

The rats were anesthetized using isoflurane (5\% to induce and $2.5-3 \%$ to maintain) and the area overlying the T7/T8 vertebrae was shaved, cleaned, and sterilized. Intravenous access via a tail vein was established for microbubbles (Definity ${ }^{\circledR}$; Lantheus, NJ, USA) and subsequent saline flushes. For acute ultrasound imaging a laminectomy was performed on thoracic level 7-9. A layer of ultrasound gel was placed directly on top of the dura and preinjury ultrasound imaging was performed. A contusion injury to the spinal cord was then performed as described above. Ultrasound imaging was again performed to image postinjury hemodynamic changes.

For noninvasive transcutaneous ultrasound imaging, hair overlying the site from the original area of laminectomy was removed. US imaging and acquisition was performed as described above and for each CEUS event, a bolus of $0.1 \mathrm{~mL}$ of microbubbles was injected intravenously followed by a $0.2 \mathrm{~mL}$ saline flush. Both cohorts of six rats were imaged transcutaneously, subcutaneously, and epiduraly through the acoustic window created by the original laminectomy surgery. Sagittal ultrasound images in the median plane were acquired by aligning the probe in the plane of the perforating median vessels. A motorized stage holding the probe was used to move the probe to sweep side to side at $50-100 \mu \mathrm{m}$ steps to determine the largest area of hypoperfusion. Multiple images were acquired along parasagittal planes. The signal-to-noise ratio (SNR) of the perfusion signal (i.e. low velocity blood flow in the microcirculation) was measured in a region of interest (ROI) in the spinal cord in two areas for each imaging approach. The first ROI was placed in a region of spinal cord clear of larger vessels in the intervening paraspinal muscle. A second ROI was placed in a region of spinal cord below a larger intervening vessel of the paraspinal muscle, to assess the effect of attenuation of overlying larger vasculature for the transcutaneous and subcutaneous acquisitions. Image reconstruction and SNR measurements were performed in MatLab (Mathworks, US).

For subcutaneous imaging, the skin overlying T5-T11 was cleaned and sterilized, and a skin incision was made. Agricola retractors held the skin back while sterile ultrasound gel was placed onto the underlying musculature. Following subcutaneous CEUS, the ultrasound gel was removed from the skin incision. Subperiostal dissection of paraspinal muscles was performed to expose the laminectomy window over the spinal cord at T7-T9. The rat was again stabilized in a custom spinal frame from the T5 and T11 vertebrae and sterile ultrasound gel was placed on top of the dura. Again, ultrasound images were captured epiduraly as described above (see Fig. 1 for illustration). The area of the hypoechoic region was measured using an image analysis tool developed in MatLab.

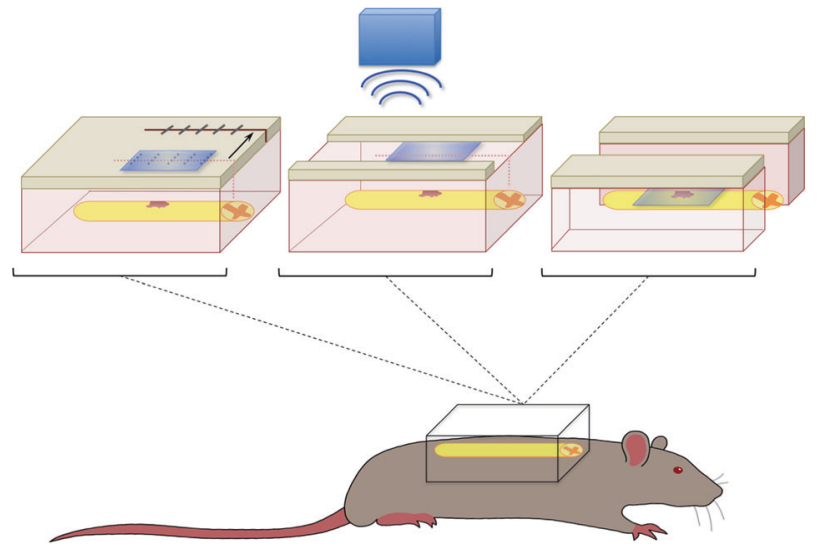

Fig. 1 Illustration of experiment setup. From left to right, representation of transcutaneous, subcutaneous, and epidural contrastenhanced ultrasound (CEUS) imaging. The transparent blue rectangle depicts the imaging window. During transcutaneous imaging, the skin overlying the incision site is gently pulled to the side to avoid the ultrasound interference of the wound closures.

\section{Histology}

At the end of the experimental period, the rats were euthanized using an overdose of Beuthanesia-D (ScheringPlough Animal Health, Kenillworth, NJ). Trans-cardiac perfusion was performed using ice cold PBS (pH 7.4, 200 $\mathrm{mL})$ followed by cold $4 \%$ paraformaldehyde $(\sim 300 \mathrm{~mL})$. The spinal cord was removed, postfixed in $4 \%$ paraformaldehyde solution overnight at $4{ }^{\circ} \mathrm{C}$ and treated with $30 \%$ sucrose solution with $0.01 \%$ sodium azide prior to freezing. Twenty micron sections were obtained using a cryostat (Leica, Wetzlar, Germany, CM1950), thaw mounted onto gelatin-coated glass slides, and stored at $-80^{\circ} \mathrm{C}$. Standard cresyl violet (labels neurons and cell nuclei) and Luxol fast blue (myelin) stainings were performed to visualize spared tissue and myelin within the spinal cord. Photomicrographs were taken with either a Zeiss Primo Star microscope with a color camera (Axiocam ERc 5s) attached, or a Leica DMI6000 inverted microscope with a color camera (Leica DFC365 FX CCD) attached. Images were processed using either Adobe Photoshop CS5.1, Adobe Illustrator CS6, or ImageJ 2.0.

\section{Area analysis}

To determine the area of hypoperfusion resulting from spinal cord injury, midsagittal perfusion CEUS images were used. All images used for perfusion deficit measurements were acquired with the same settings of the Verasonics system. At 3 days post injury $(n=6)$, the area of perfusion deficit was measured using the freehand selection tool in ImageJ (ImageJ 2.0; Wayne Rasband, retired from NIH). At 10-week post injury $(n=6)$ multiple smaller 
Fig. 2 CEUS imaging detects tissue perfusion and vascular flow within the spinal cord in a rodent. CEUS imaging was performed before and immediately after a contusion type SCI in a rat. Tissue perfusion $(\mathbf{a}, \mathbf{c})$ and vascular flow $(\mathbf{b}, \mathbf{d})$ from intact and injured spinal cord were imaged immediately after injury.
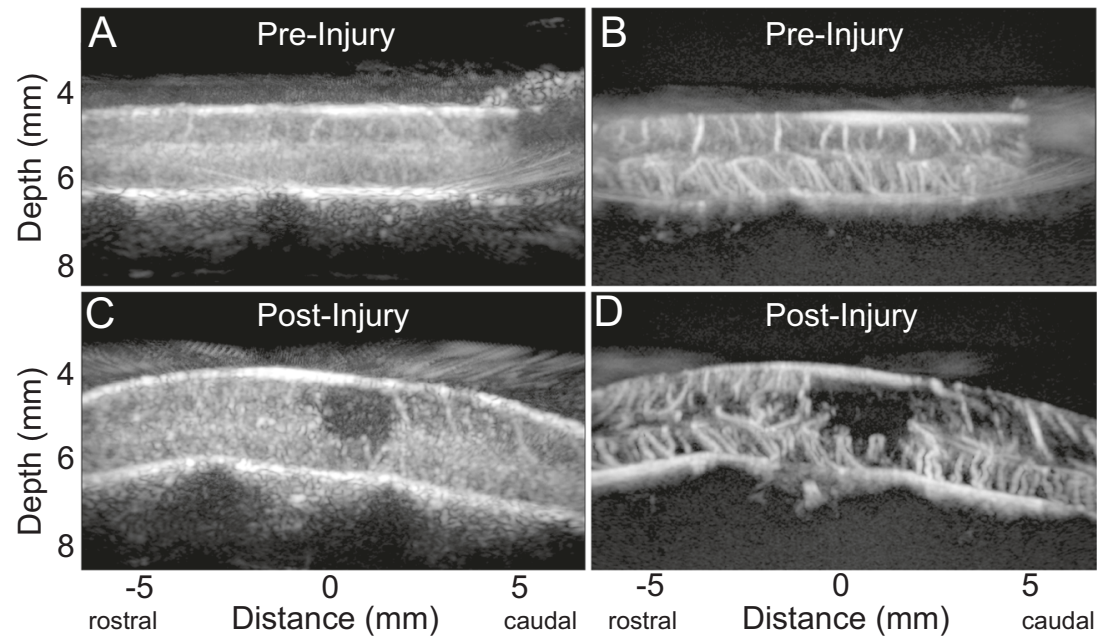

$\left(\leq 1 \mathrm{~mm}^{2}\right)$ cystic regions were observed. For this group, the largest cystic regions of each rat were measured (see example in Fig. 5) with the same CEUS method. To correlate perfusion deficits detected via ultrasound imaging with histological tissue sparing, a $3 \mathrm{~mm}$ portion of the cord spanning the lesion center was analyzed. This window reliably contained both the perfusion deficits seen with CEUS, and the cystic regions observed histologically. The total area of the spinal cord within this $3 \mathrm{~mm}$ section was measured for both histological samples and ultrasound images. Then, hypoechoic regions were measured for ultrasound images, and cystic cavities were measured for histological samples. In order to approximate the volume of healthy tissue for both approaches, the volumes of hypoechoic regions and cystic regions were subtracted from the total volume taken by the spinal cord. We chose this method over directly comparing the volumes of hypoechoic regions and cystic cavities because cystic cavities tended to collapse during tissue fixation and cyro sectioning.

In order to verify that measurements of hypoechoic regions were consistent between and within raters, three separate raters measured the hypoechoic regions of five midsagittal perfusion ultrasound images acquired from animals who had received $200 \mathrm{kD}$ injuries. The hypoperfused areas ranged in size from 0.3 to $3 \mathrm{~mm}^{2}$. Each rater scored the same set of images on three separate occasions. Ordinary two-way ANOVA tests were performed for each rater's individual scores, as well as for all raters using averaged scores. Intraclass correlation coefficients (ICCs) were calculated following a two-way mixed effects consistency model, following the guidelines outlined by McGraw and Wong [24]. The ICC comparing the scores of all raters was 0.979 , and individual ICCs ranged from 0.974 to 0.999 . These scores fall within the range generally accepted as "excellent reliability". Hypoperfusion measurements presented here were collected by a single rater.

\section{Statistical analysis}

Continuous variables are depicted as means \pm standard error of the mean. GradPad Prism 7 (GraphPad Software, Inc., San Diego, CA) was used to perform all statistical analyses. Measured areas of perfusion deficits from CEUS images were analyzed using ANOVA analysis of variance, and the significance was set at $p=0.05$.

\section{Results}

CEUS allowed for acquisition of sagittal images of the injured spinal cord via the acoustic window of the laminectomy. Figure 2 shows CEUS images of tissue perfusion and vascular images before injury $(\mathrm{a}, \mathrm{b})$ and immediately after injury $(\mathrm{c}, \mathrm{d})$. The spinal cord blood flow was again visualized in animals at 3 days post injury through the skin (Fig. 3a, b), through the muscle layers subcutaneously (Fig. 3c, d), and epidurally (Fig. 3e, f). A progressive loss in the signal amplitude was observed when imaging was carried out through the attenuative layers of the skin and musculature for both the vascular flow and perfusion images. Table 1 shows the decrease in spinal cord perfusion signal of transcutaneous and subcutaneous CEUS imaging compared with epidural imaging $(n=6)$. An average loss of $7.2 \mathrm{~dB}$ and $5.1 \mathrm{~dB}$ in SNR was measured when imaging transcutaneously and subcutaneously compared with epidural imaging. An additional 3-4 dB signal loss in the spinal cord was observed when shadowed by a larger vessel in paraspinal muscle tissue. At 3 days post injury, area measurements of the hypoperfused injury center were similar when comparing transcutaneous, subcutaneous, and epidural ultrasound acquisitions $(5.78 \pm 0.86,5.91 \pm$ $\left.0.53,5.65 \pm 1.07 \mathrm{~mm}^{2} ; p>0.05\right)$. 
Fig. 3 CEUS imaging performed through the acoustic window subacutely. Change in tissue perfusion (low velocity microbubble signal) and vascular flow (high velocity microbubble signal) were imaged transcutaneously, subcutaneously or epidurally at 3 days following injury $(N=6)$. Example images of hypoperfused area (a transcutaneous, c subcutaneous, e epidural) and vascular flow changes are shown (b transcutaneous, $\mathbf{d}$ subcutaneous, f epidural). The dynamic range for the vascular flow and perfusion images were 40 and $30 \mathrm{~dB}$, respectively.
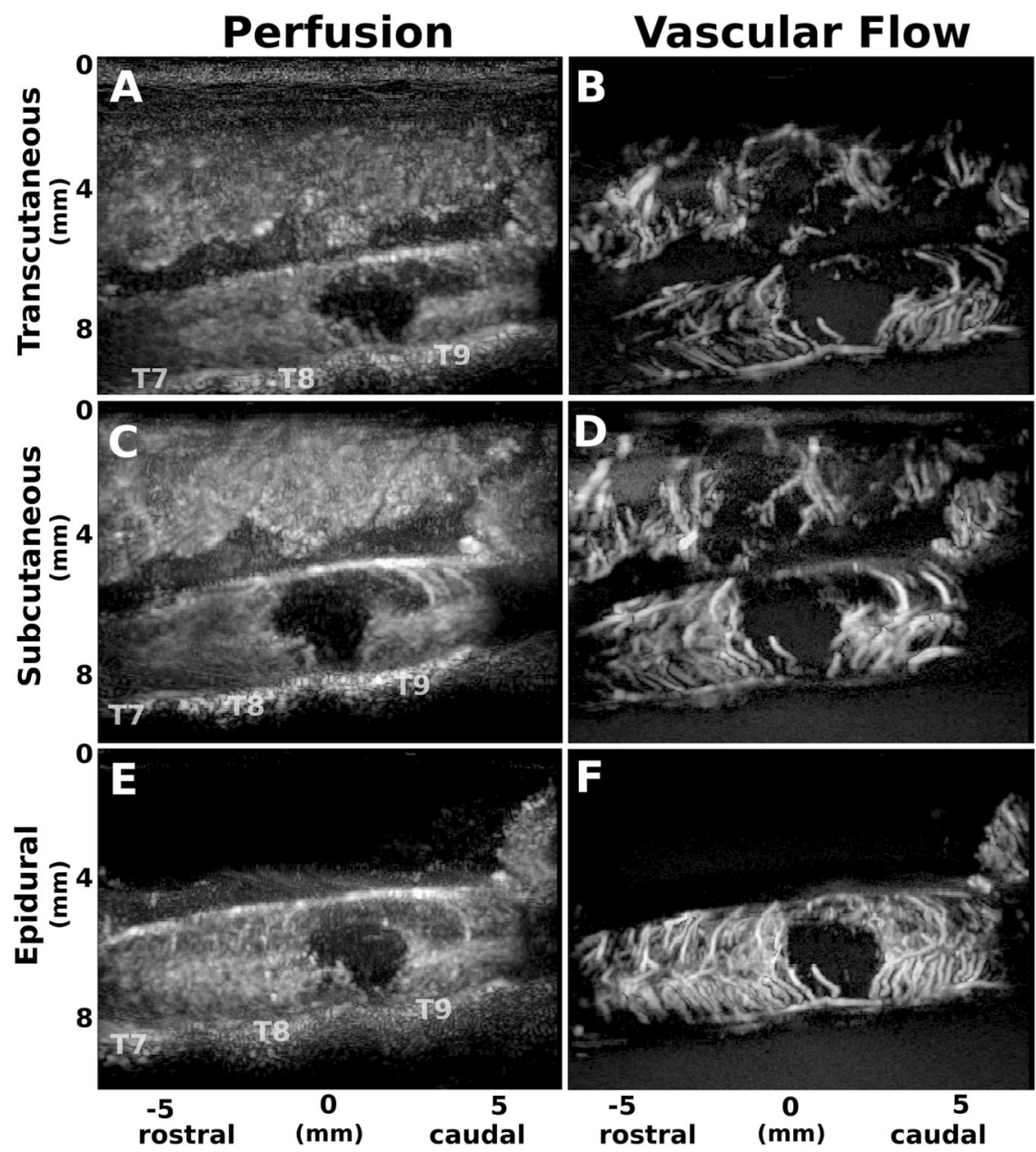

Table 1 Acquisition dependent CEUS characteristics.

\begin{tabular}{ll}
\hline Imaging approach & Signal-to-noise ratio \\
\hline Transcutaneous & $19.3 \pm 2.5 \mathrm{~dB}$ \\
Subcutaneous & $26.5 \pm 2.2 \mathrm{~dB}$ \\
Epidural & $31.6 \pm 3.2 \mathrm{~dB}$ \\
\hline
\end{tabular}

CEUS imaging at 10 weeks after injury revealed expected spinal cord atrophy and a small remaining area of hypoperfusion (Fig. 4). The dynamic range of the perfusion and vascular flow images was $40 \mathrm{~dB}$ and $30 \mathrm{~dB}$, respectively. The perfusion image revealed a small hypoechoic region in the center of the spinal cord and a atrophy of the of spinal cord extending beyond the original contusion site. The hypoechoic region was at the edge of a cyst (determined using b-mode ultrasound images; data not shown), which was centered more in the lateral aspect of the spinal cord. No statistically significant differences in the area measures of the largest hypoperfused regions were observed, when measured transcutaneously, subcutaneously or epidurally $\left(0.69 \pm 0.05,1.09 \pm 0.11,0.95 \pm 0.11 \mathrm{~mm}^{2}\right.$, respectively; $p>0.05$ ) at 10 weeks post injury. The vascular flow images depict morphological changes in vessel orientation and tortuosity extending beyond the primary injury site.

In summary, statistically similar areas of hypoechoic regions at each time point ( 3 days and 10 weeks following injury) were measured regardless of whether the images were transcutaneously, subcutaneously, or epidurally acquired (Fig. 5). There was a significant decrease in the hypoperfused area detected at 10 weeks compared with 3 days post injury $(p<0.001)$ suggesting that CEUS imaging (all three levels) were able to detect significant alterations in the spinal tissue and vasculature with time after injury. We have also examined the functional deficits in SCI animals with and without chronic repeated ultrasound imaging, and showed that there were no statistical differences in their open-field locomotor score (Basso, Beatie, Bresnahan (BBB) score; Fig. 5d) suggesting that the multiple imaging sessions did not alter their functional recovery after SCI.

At chronic time points, multiple cystic regions were observed, accompanied by a reduction in spinal cord diameter (Fig. 6a) concordant with our previous experience as 
Fig. 4 CEUS imaging performed through the original acoustic window chronically. Change in tissue perfusion (low-velocity microbubble signal) and vascular flow (high-velocity microbubble signal) were imaged transcutaneously, subcutaneously at 10 weeks following injury $(N=6)$. Example images of hypoperfused area (a transcutaneous, c subcutaneous, e epidural) and vascular flow changes are shown (b transcutaneous, $\mathbf{d}$ subcutaneous, f epidural). The dynamic range for the vascular flow and perfusion images were 40 and $30 \mathrm{~dB}$, respectively.
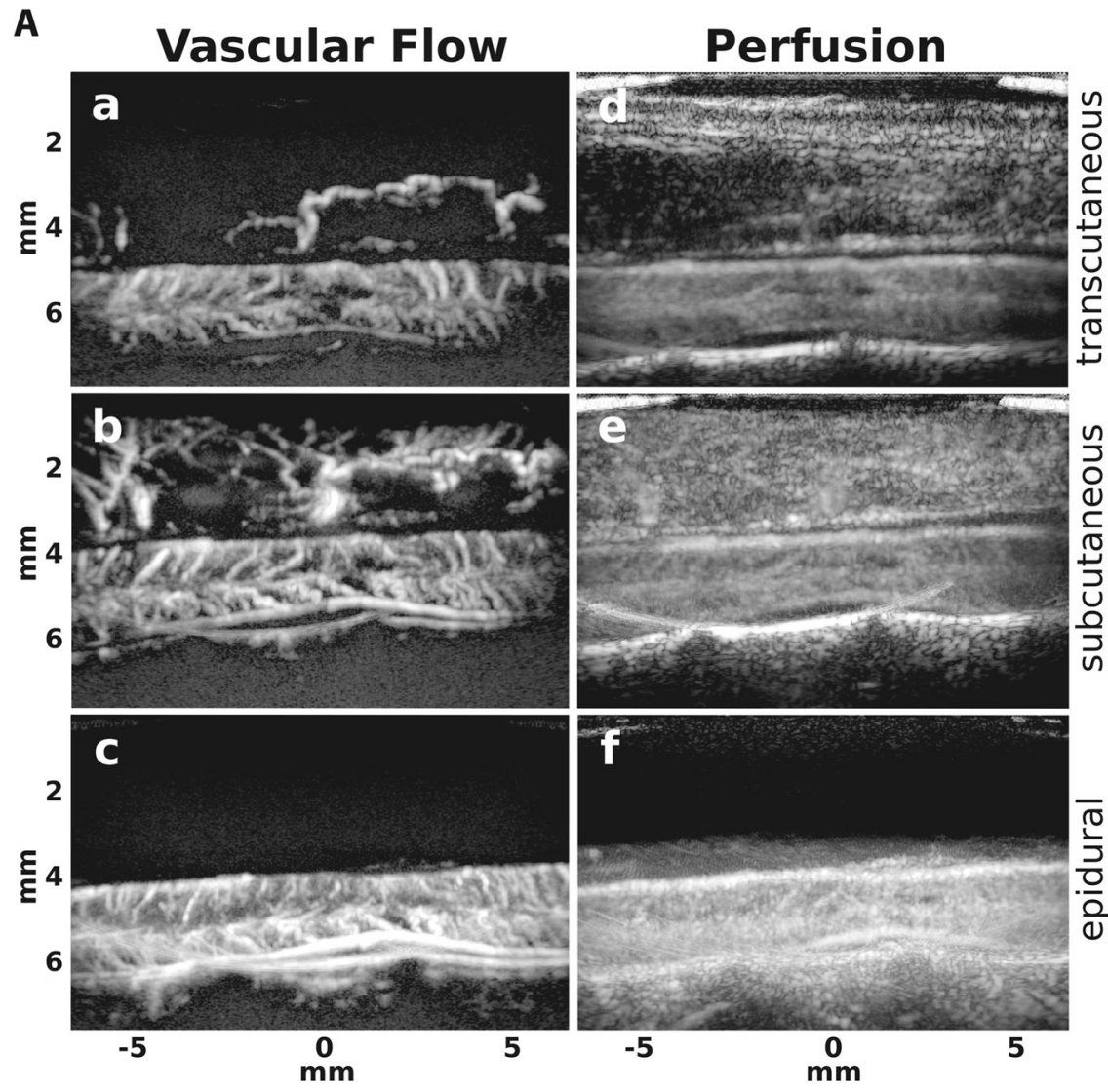

well as other published work on this model of SCI [25]. We correlated the intact tissue detected at lesion center $(3 \mathrm{~mm}$ wide) observed from histologically stained sections and compared them to intact perfusion tissue detected using ultrasound imaging. We found that there was a statistically significant positive correlation $\left(r^{2}=0.70, p<0.02\right.$; Fig. 6b), suggesting that areas with intact perfusion on ultrasound images correspond to intact tissue detected by the gold-standard histological staining.

\section{Discussion}

In the current report, we demonstrate that transcutaneous CEUS imaging of the contused rodent spinal cord is feasible. Importantly, despite signal attenuation caused by the overlying skin and paraspinal tissue, transcutaneous CEUS measurements allow for estimation of local tissue hypoperfusion and thus noninvasive longitudinal assessment of blood flow changes following experimental tSCI. Although some ultrasound signal attenuation was experienced in imaging through intervening microbubbles and paraspinal tissue, similar views of the intrinsic spinal cord vasculature and resulting deficits following tSCI were observed for all three imaging approaches (i.e., transcutaneous, subcutaneous and epidural). A mean decrease of $5.1 \mathrm{~dB}$ in perfusion signal was observed when imaging subcutaneously compared to directly over the spinal cord (epidurally), with an additional mean loss of $7.2 \mathrm{~dB}$ observed when imaging through both paraspinal muscle and skin. To assess the potential differences of the imaging approaches, we compared the measurements of the area of perfusion deficit following SCI for each imaging approach at two time points (acute and 10 weeks). We found similar area measurements for each approach at both time points suggesting that transcutaneous CEUS imaging technique could be useful to noninvasively monitor blood flow changes following tSCI in a rodent model.

Given the impact of spinal cord blood perfusion on functional outcome after traumatic injury [26], multiple different monitoring technologies have been developed. Measurements of intraspinal pressure allow for calculation of spinal perfusion pressure as well as estimation of the optimum spinal perfusion pressure [15]. We and others have found that traumatic SCI leads to a threefold elevation of intraspinal pressure at the injury site compared with physiological pressures and pressures remain elevated for up to 7 days in rodents [12], pigs [27], and humans [9]. Importantly, measured tissue perfusion positively correlates with amplitudes of motor evoked potentials (9/9 patients tested) [9]. Intraspinal pressure monitoring has shown promise in the management of patients with acute spinal cord injury 
A

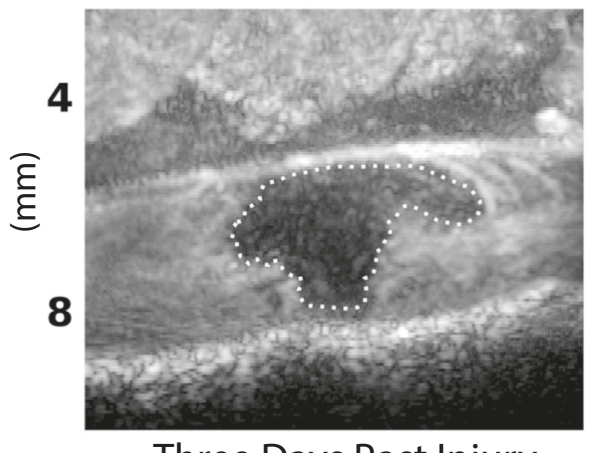

Three Days Post Injury

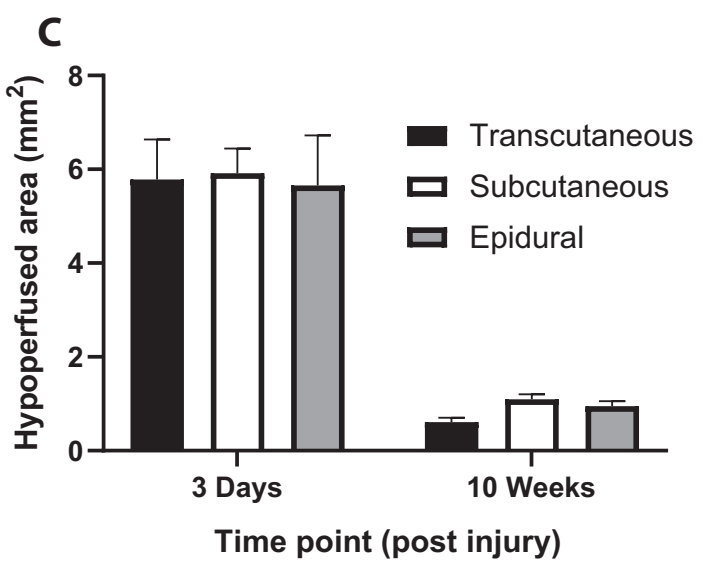

Fig. 5 Transcutaneous CEUS imaging detects area of tissue perfusion deficit after SCI. a, b Example of hypoperfused areas at subacute (3 days) and chronic (10 weeks) time points after SCI. c Significantly smaller area of tissue hypoperfusion was detected at 10 weeks compared with 3 days post injury $(* p<0.001)$. No significant differences in areas were detected when CEUS imaging was

$[9,10]$. While technically feasible and safe, this requires intradural implantation of a pressure sensor which provides measurements at only one location [28]. Therefore, direct measurements of hemodynamic changes within the spinal cord tissue after SCI is needed.

Near infrared spectroscopy constitutes another promising technology for monitoring spinal cord hemodynamics (for an excellent review, please see [15]). Near infrared spectroscopy allows for the measurements of tissue oxygen saturation in real time [29] and is approved for continuous monitoring of brain oxygen saturation [30]. While near infrared spectroscopy cannot reliably measure spinal cord hemodynamics via transcutaneous acquisition [31], accurate epidural measurements have been demonstrated in an experimental porcine spinal cord injury model [15]. In these experiments near infrared spectroscopy accurately measured spinal cord tissue oxygenation in response to hypoxia and changes of the mean arterial pressure. Potential limitations of near infrared spectroscopy are poor spatial resolution as

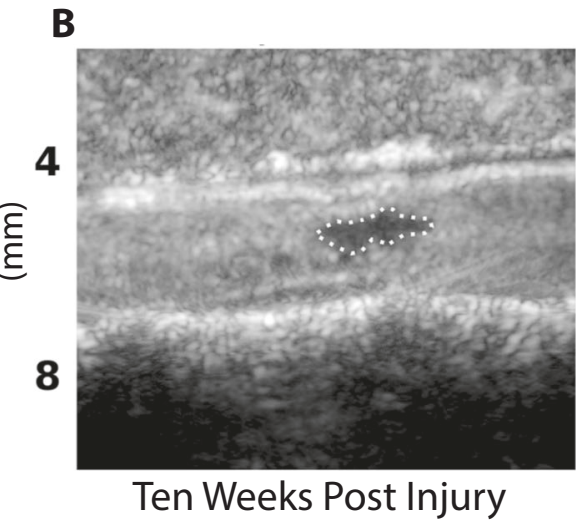

D

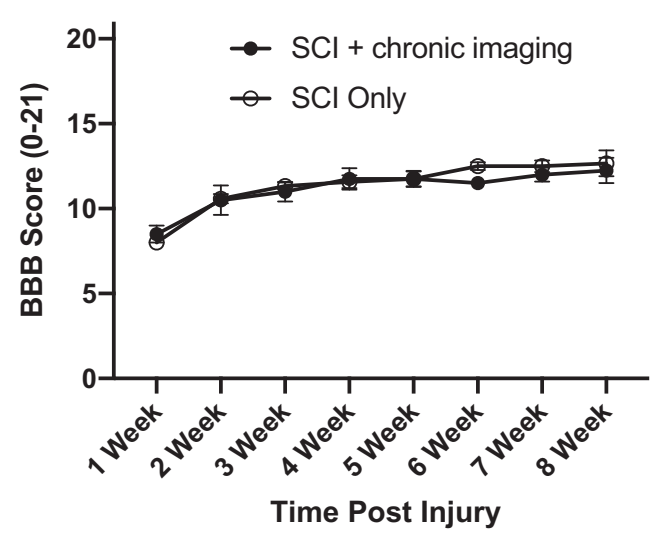

performed either transcutaneously or subcutaneously compared with epidurally. d Weekly Basso, Beatie and Bresnahan open-field locomotor testing of rats after spinal cord injury, with (closed circles; $N=$ 6) and without (open circles; $N=5$ ) showed that there were no functional differences in their hindlimb function for up to 8 week post injury $(p>0.05)$.

the pathlength taken by the photons through the spinal cord tissue is unknown, the low SNR and possible thermal tissue damage [15].

Laser Doppler flowmetry utilizes the Doppler shift that light undergoes when it is reflected by moving red blood cells [32]. This technology has been used to assess local spinal cord blood flow in various spinal cord injury models [33-36]. Laser Doppler is typically collected epidurally and provides real-time blood flow estimates. However, laser Doppler delivers a volume-averaged estimate of blood flow beneath the probe limited to a depth of $\sim 1 \mathrm{~mm}$. Moreover, local swelling of contused spinal cord tissue and the resulting changes of cerebrospinal fluid (CSF) space and CSF pulsations may explain some of the contradicting results [34] compared traditional hydrogen clearance [37] or microsphere deposition techniques [38].

Ultrasound imaging of the spinal cord is well established. Initial studies used B-mode ultrasound imaging to visualize posttraumatic microstructural spinal cord abnormalities in 
A

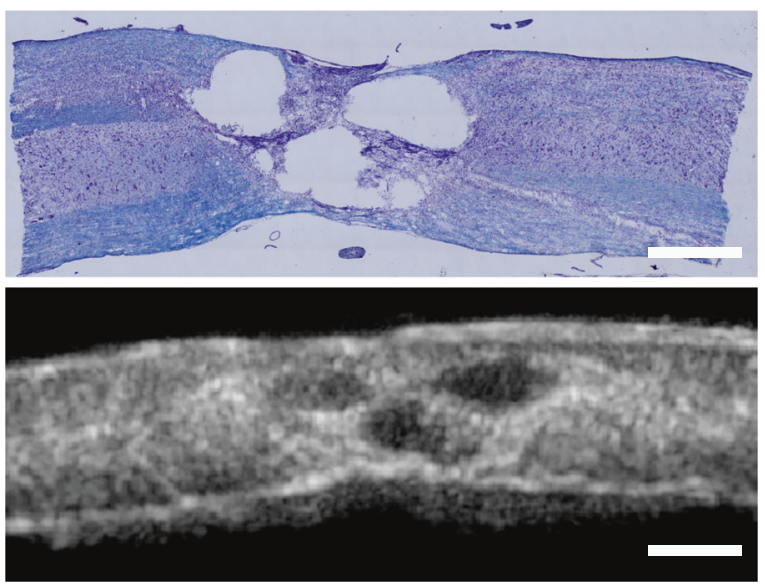

B

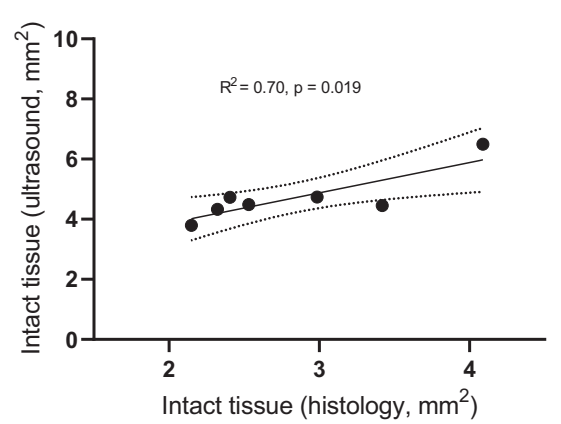

Fig. 6 Extent of spared tissue estimated with CEUS imaging correlates with histological measurements. a Thin longitudinal section of a spinal cord at 10 weeks post SCI was labeled with Nissl staining and the same spinal cord was imaging using CEUS imaging. Scale bar $=1 \mathrm{~mm}$. b Correlation of intact tissue in a $3 \mathrm{~mm}$ long segment of the spinal cord using histology and CEUS imaging $(N=7)$.

rodents [17, 21], pigs [39], and patients [40]. Indeed, the Fehlings' group has utilized ultrasound imaging (B-mode and power Doppler imaging) to examine the vascular changes after SCI after multiple treatment paradigms [41, 42], suggesting that ultrasound imaging is a useful technique for examining blood flow changes within the entire spinal cord after SCI.

Utilizing intravenous ultrasound contrast in combination with traditional ultrasound, Soubeyrand et al. first demonstrated hemodynamic alterations in a rodent SCI model [18]. CEUS imaging confirmed a dramatic hypoperfusion at the center of a T10 contusion injury [18]. Importantly, hypoperfusion of the lesion center and penumbra worsened during the first hour after trauma, indicating that this technology might be well suited to monitor and guide interventions treating local ischemia. However, the sensitivity and spatial resolution of CEUS in this study was limited by the utilization of a conventional ultrasound system architecture (Visualsonics).

High ultrasound frequencies are necessary to achieve high spatial resolution of acquired images. However, previous
CEUS imagining techniques were limited at elevated frequencies $(>7 \mathrm{MHz})$ due to the diminishing microbubble response beyond their resonance frequency $(1-2 \mathrm{MHz})$ [43]. We have overcome this limitation using a high framerate plane-wave (i.e. ultrafast) imaging approach that overcomes the weak microbubble response by essentially accumulating multiple frames. In addition, our acquisition enables Doppler processing to extract higher velocity microbubble flow $(\sim 0.5-1 \mathrm{~cm} / \mathrm{s})$ in the larger vasculature with advantages in sensitivity over conventional Doppler [44]. This approach overcomes the challenges of imaging microbubbles at elevated frequencies and spatial resolutions necessary to image rodent models [20, 21]. Microbubbles give ultrasound the ability to image low velocity $(\sim \mathrm{mm} / \mathrm{s})$ blood flow in the microcirculation beyond the limitations of Doppler ultrasound. Utilizing a high frequency CEUS approach thus enables distinction of low velocity microbubbles in the microcirculation from higher velocity microbubbles in larger vasculature [22, 23, 44]. Utilizing this strategy, ultrasound signal obtained from microvasculature that facilitates exchange of oxygen, electrolytes and nutrients is only minimally affected by the signal from larger vessels. This technology provides a topographical map of local tissue hypoperfusion with high sensitivity and spatial resolution. It allows detection and quantification of spinal cord tissue in the penumbra of the injury center suffering from a critical loss of perfusion [21]. However, similar to all other aforementioned techniques for in vivo spinal cord blood flow monitoring CEUS has been only obtained with the ultrasound transducer positioned in the epidural space $[17,18,21]$. Thus, opening of the skin, subcutaneous tissue, paraspinal muscles and laminae were required. In the current report, we demonstrate for the first time the feasibility to acquire CEUS imaging of the posttraumatic spinal cord in rodents transcutaneously via the acoustic window created by the original laminectomy. Moreover, transcutaneous CEUS imaging provided similar area measurements of hypoperfused regions compared with subcutaneous or epidural imaging, despite signal attenuation from intervening paraspinal muscles and vasculature. Thus, our current study suggests that transcutaneous CEUS imaging technique constitutes a feasible strategy to estimate spinal cord tissue perfusion in a rodent spinal cord injury model.

Decompression and stabilization of the spinal column is recommended within $24 \mathrm{~h}$ after injury and routinely performed at our level one trauma center [4-7]. Almost all patients with any remaining neurological function undergo posterior decompression and stabilization surgeries which provide an acoustic window to the contused spinal cord. Therefore, conventional CEUS or more advanced CEUS approaches could potentially be developed for noninvasive monitoring of spinal cord blood flow and perfusion as part of the management of spinal cord injured patients. 
We acknowledge that our current study has several limitations. While transcutaneous ultrasound B-mode imaging of the human spinal cord via the acoustic window of a laminectomy has been demonstrated $[45,46]$, the feasibility to translate our initial rodent results into the clinic remains to be determined. Moreover, transcutaneous CEUS was obtained during a terminal ultrasound session in anesthetized animals, rather than as continuous monitoring in awake animals. In addition, 3D scans across the entire spinal cord volume are needed to truly assess the volume of the hypoperfused spinal cord regions after injury.

\section{Conclusion}

In conclusion, transcutaneous CEUS is the first demonstration of noninvasive measurements and monitoring of local hemodynamic changes in a rodent traumatic SCI model.

\section{Data availability}

The datasets generated during this study are available from the corresponding author on reasonable request.

Funding This work was supported by awards from the Craig Neilsen Foundation, DoD CDMRP Translational Award (W81XWH-18-10753), and Department of Neurological Surgery.

Author contributions $\mathrm{ZZK}, \mathrm{MB}$ and $\mathrm{CPH}$ designed experiments, $\mathrm{ZZK}, \mathrm{MB}$ and $\mathrm{LNC}$ performed experiments and recorded data, ZZK, MB, LNC, JEH and RH analyzed data, ZZK, MB and CPH wrote the paper and all authors reviewed and approved the paper.

\section{Compliance with ethical standards}

Conflict of interest The authors declare that they have no conflict of interest.

Ethics All work performed in this study was approved by the Institutional Animal Care and Use Committee (IACUC) at the University of Washington (Protocol Number: 4362-01). We certify that all applicable institutional and governmental regulations concerning the ethical use of vertebrate animals were followed during the course of this research.

Publisher's note Springer Nature remains neutral with regard to jurisdictional claims in published maps and institutional affiliations.

\section{References}

1. Ducker TB, Assenmacher DR. Microvascular response to experimental spinal cord trauma. Surg Forum. 1969;20:428-30.

2. Guha A, Tator CH, Rochon J. Spinal cord blood flow and systemic blood pressure after experimental spinal cord injury in rats. Stroke. 1989;20:372-7.
3. Mautes AE, Weinzierl MR, Donovan F, Noble LJ. Vascular events after spinal cord injury: contribution to secondary pathogenesis. Phys Ther. 2000;80:673-87.

4. Fehlings MG, Vaccaro A, Wilson JR, Singh A, WC D, Harrop JS, et al. Early versus delayed decompression for traumatic cervical spinal cord injury: results of the Surgical Timing in Acute Spinal Cord Injury Study (STASCIS). PloS ONE. 2012;7:e32037.

5. Furlan JC, Noonan V, Cadotte DW, Fehlings MG. Timing of decompressive surgery of spinal cord after traumatic spinal cord injury: an evidence-based examination of pre-clinical and clinical studies. J Neurotrauma. 2011;28:1371-99.

6. Wilson JR, Singh A, Craven C, Verrier MC, Drew B, Ahn H, et al. Early versus late surgery for traumatic spinal cord injury: the results of a prospective Canadian cohort study. Spinal Cord. 2012;50:840-3.

7. Wyndaele JJ. The impact of early versus late surgical decompression on neurological recovery after traumatic spinal cord injury (SCI). Spinal Cord. 2012;50:789.

8. Walters BC, Hadley MN, Hurlbert RJ, Aarabi B, Dhall SS, Gelb $\mathrm{DE}$, et al. Guidelines for the management of acute cervical spine and spinal cord injuries: 2013 update. Neurosurgery 2013;60 (Suppl 1):82-91.

9. Werndle MC, Saadoun S, Phang I, Czosnyka M, Varsos GV, Czosnyka $\mathrm{ZH}$, et al. Monitoring of spinal cord perfusion pressure in acute spinal cord injury: initial findings of the injured spinal cord pressure evaluation study. Crit Care Med. 2014;42:646-55.

10. Phang I, Papadopoulos MC. Intraspinal pressure monitoring in a patient with spinal cord injury reveals different intradural compartments: injured spinal cord pressure evaluation (ISCoPE) study. Neurocrit Care. 2015;23:414-8.

11. Inoue T, Manley GT, Patel N, Whetstone WD. Medical and surgical management after spinal cord injury: vasopressor usage, early surgerys, and complications. J Neurotrauma. 2014;31: 284-91.

12. Khaing ZZ, Cates LN, Fischedick AE, McClintic AM, Mourad PD, Hofstetter CP. Temporal and spatial evolution of raised intraspinal pressure following traumatic spinal cord injury. J Neurotrauma. 2017;34:645-51.

13. Khaing ZZ, Ehsanipour A, Hofstetter CP, Seidlits SK. Injectable hydrogels for spinal cord repair: a focus on swelling and intraspinal pressure. Cells Tissues Organs. 2016;202:67-84.

14. Kogler AS, Bilfinger TV, Galler RM, Mesquita RC, Cutrone M, Schenkel SS, et al. Fiber-optic monitoring of spinal cord hemodynamics in experimental aortic occlusion. Anesthesiology. 2015;123:1362-73.

15. Rashnavadi T, Macnab A, Cheung A, Shadgan A, Kwon BK, Shadgan B. Monitoring spinal cord hemodynamics and tissue oxygenation: a review of the literature with special focus on the near-infrared spectroscopy technique. Spinal Cord. 2019;57:617-25.

16. Busch DR, Davis J, Kogler A, Galler RM, Parthasarathy AB, Yodh AG, et al. Laser safety in fiber-optic monitoring of spinal cord hemodynamics: a preclinical evaluation. J Biomed Opt. 2018;23:1-9.

17. Soubeyrand M, Badner A, Vawda R, Chung YS, Fehlings MG. Very high resolution ultrasound imaging for real-time quantitative visualization of vascular disruption after spinal cord injury. $\mathrm{J}$ Neurotrauma. 2014;31:1767-75.

18. Soubeyrand M, Laemmel E, Dubory A, Vicaut E, Court C, Duranteau J. Real-time and spatial quantification using contrastenhanced ultrasonography of spinal cord perfusion during experimental spinal cord injury. Spine. 2012;37:E1376-82.

19. Dubory A, Laemmel E, Badner A, Duranteau J, Vicaut E, Court $\mathrm{C}$, et al. Contrast enhanced ultrasound imaging for assessment of spinal cord blood flow in experimental spinal cord injury. J Vis Exp. 2015;7:e52536. 
20. Bruce M, Hannah A, Hammond R, Khaing ZZ, TremblayDarveau C, Burns PN, et al. High frequency nonlinear Doppler contrast-enhanced ultrasound imaging of blood flow. IEEE Trans Ultrason Ferroelectr Freq Control. 2020, in press.

21. Khaing ZZ, Cates LN, DeWees DM, Hannah A, Mourad P, Bruce $\mathrm{M}$, et al. Contrast-enhanced ultrasound to visualize hemodynamic changes after rodent spinal cord injury. J Neurosurg Spine. 2018;29:306-13.

22. Tremblay-Darveau C, Williams R, Milot L, Bruce M, Burns $\mathrm{PN}$. Combined perfusion and doppler imaging using planewave nonlinear detection and microbubble contrast agents. IEEE Trans Ultrason Ferroelectr Freq Control. 2014;61: 1988-2000

23. Tremblay-Darveau C, Williams R, Sheeran PS, Milot L, Bruce M, Burns PN. Concepts and tradeoffs in velocity estimation with plane-wave contrast-enhanced Doppler. IEEE Trans Ultrason Ferroelectr Freq Control. 2016;63:1890-905.

24. McGraw KO, Wong SP. Forming inferences about some intraclass correlation coefficients. Psychol Methods. 1996;1:30-46.

25. Cao Q, Zhang YP, Iannotti C, DeVries WH, Xu XM, Shields CB, et al. Functional and electrophysiological changes after graded traumatic spinal cord injury in adult rat. Exp Neurol. 2005;191 Suppl 1:S3-S16.

26. Tator $\mathrm{CH}$, Fehlings MG. Review of the secondary injury theory of acute spinal cord trauma with emphasis on vascular mechanisms. J Neurosurg. 1991;75:15-26.

27. Guest JD, Moore SW, Aimetti AA, Kutikov AB, Santamaria AJ, Hofstetter CP, et al. Internal decompression of the acutely contused spinal cord: differential effects of irrigation only versus biodegradable scaffold implantation. Biomaterials. 2018;185: 284-300.

28. Chen S, Smielewski P, Czosnyka M, Papadopoulos MC, Saadoun S. Continuous monitoring and visualization of optimum spinal cord perfusion pressure in patients with acute cord injury. J Neurotrauma. 2017;34:2941-9.

29. Jobsis FF. What is a molecular oxygen sensor? What is a transduction process? Adv Exp Med Biol. 1977;78:3-18.

30. Murkin JM. NIRS: a standard of care for CPB vs. an evolving standard for selective cerebral perfusion? J Extra Corpor Technol. 2009;41:P11-4.

31. Suehiro K, Funao T, Fujimoto Y, Mukai A, Nakamura M, Nishikawa K. Transcutaneous near-infrared spectroscopy for monitoring spinal cord ischemia: an experimental study in swine. J Clin Monit Comput. 2017;31:975-9.

32. Nilsson GE, Tenland T, Oberg PA. Evaluation of a laser Doppler flowmeter for measurement of tissue blood flow. IEEE Trans Biomed Eng. 1980;27:597-604.

33. Carlson GD, Gorden CD, Nakazawa S, Wada E, Smith JS, LaManna JC. Sustained spinal cord compression: part II: effect of methylprednisolone on regional blood flow and recovery of somatosensory evoked potentials. J Bone Jt Surg Am. 2003; 85:95-101.

34. Horn EM, Theodore N, Assina R, Spetzler RF, Sonntag VK, Preul MC. The effects of intrathecal hypotension on tissue perfusion and pathophysiological outcome after acute spinal cord injury. Neurosurg Focus. 2008;25:E12.

35. Hamamoto Y, Ogata T, Morino T, Hino M, Yamamoto H. Realtime direct measurement of spinal cord blood flow at the site of compression: relationship between blood flow recovery and motor deficiency in spinal cord injury. Spine. 2007;32:1955-62.

36. Westergren H, Farooque M, Olsson Y, Holtz A. Spinal cord blood flow changes following systemic hypothermia and spinal cord compression injury: an experimental study in the rat using LaserDoppler flowmetry. Spinal Cord. 2001;39:74-84.

37. Griffiths IR, Rowan JO, Crawford RA. Spinal cord blood flow measured by a hydrogen clearance technique. J Neurol Sci. 1975;26:529-44.

38. Elmore JR, Gloviczki P, Harper CM,Jr, Murray MJ, Wu QH, Bower TC, et al. Spinal cord injury in experimental thoracic aortic occlusion: investigation of combined methods of protection. $\mathrm{J}$ Vasc Surg. 1992;15:789-98.

39. Santamaria AJ, Benavides FD, Padgett KR, Guada LG, NunezGomez Y, Solano JP, et al. Dichotomous locomotor recoveries are predicted by acute changes in segmental blood flow after thoracic spinal contusion injuries in pigs. J Neurotrauma. 2019;36: $1399-415$

40. Quencer RM. The injured spinal cord. Evaluation with magnetic resonance and intraoperative sonography. Radio Clin N Am. 1988;26:1025-45.

41. Badner A, Vawda R, Laliberte A, Hong J, Mikhail M, Jose A, et al. Early intravenous delivery of human brain stromal cells modulates systemic inflammation and leads to vasoprotection in traumatic spinal cord injury. Stem Cells Transl Med. 2016;5:991-1003.

42. Badner A, Vidal PM, Hong J, Hacker J, Fehlings MG. Endogenous Interleukin-10 deficiency exacerbates vascular pathology in traumatic cervical spinal cord injury. J Neurotrauma. 2019;36: 2298-307.

43. Goertz DE, Cherin E, Needles A, Karshafian R, Brown AS, Burns $\mathrm{PN}$, et al. High frequency nonlinear B-scan imaging of microbubble contrast agents. IEEE Trans Ultrason Ferroelectr Freq Control. 2005;52:65-79.

44. Tremblay-Darveau C, Williams R, Milot L, Bruce M, Burns PN. Visualizing the tumor microvasculature with a nonlinear planewave doppler imaging scheme based on amplitude modulation. IEEE Trans Med Imaging. 2016;35:699-709.

45. Mende U, Zoller J, Drommer R, Born IA, Poepel B. Realtime sonography. ZWR 1989;98:526-32.

46. Horii SC, Raghavendra BN. Transcutaneous sonography of the postoperative spine. Neuroradiology. 1986;28:599-607. 